\title{
Exact Results for Thermodynamics of the Classical Field Theories: Sine- and Sinh-Gordon Models
}

\author{
Emiliano Papa and Alexei M. Tsvelik \\ Department of Theoretical Physics \\ University of Oxford \\ 1 Keble Road \\ Oxford OX1 3NP
}

\begin{abstract}
Using the recently obtained exact results for the expectation values of operators in the sine- and sinh-Gordon models [A. B. Zamolodchikov and S. Lukyanov, Nucl. Phys. B493, 571 (1997), V. Fateev, S. Lukyanov, A. B. Zamolodchikov and Al. B. Zamolodchikov, Phys. Lett. B406, 83 (1997)] we calculate the specific heat of the corresponding two dimensional Euclidean (classical) models. We show that the temperature dependence of the specific heat of the sine-Gordon model, in the commensurate phase, has a maximum well below the Kosterlitz-Thouless transition and that the sinh-Gordon model is thermodynamically unstable in the strong coupling regime. We give also the temperature dependence of the specific heat in the incommensurate phase of the sine-Gordon model.

PACS numbers: 65.40.-f, 65.50.+m
\end{abstract}




\section{INTRODUCTION}

The sine-Gordon model is an exactly solvable model which has an enormous number of applications in condensed matter physics and statistical mechanics. It has been studied for years with many remarkable results being obtained. However, most of the effort has been concentrated on studying this model as a quantum field theory. In this paper we discuss the sine-Gordon model together with a less famous sinh-Gordon model as models of classical statistical mechanics analyzing the behavior of their specific heat in the various range of parameters.

Let us consider the classical sine- and sinh-Gordon models whose partition functions are given by

$$
\begin{aligned}
Z & =\int \mathcal{D} \varphi e^{-S[\varphi]}, \\
S_{\mathrm{sin}} & \equiv \frac{1}{T} E_{\sin }[\varphi]=\int d^{2} x\left[\frac{\rho_{\mathrm{s}}}{2 T}|\nabla \varphi-\mathrm{Q}|^{2}+\frac{m}{T}(1-\cos \varphi)\right], \\
S_{\mathrm{sinh}} & \equiv \frac{1}{T} E_{\sinh }[\varphi]=\int d^{2} x\left[\frac{\rho_{\mathrm{s}}}{2 T}|\nabla \varphi|^{2}+\frac{m}{T}(\cosh \varphi-1)\right] .
\end{aligned}
$$

The first model describes, for example, the commensurate-incommensurate transition [1]. Most recently it has been applied to double-layered Quantum Hall systems [2].

The incommensurate phase appears when $|\mathbf{Q}|$ exceeds some critical value and is characterized by nonzero average value $\langle\mathbf{Q} \nabla \varphi\rangle$. Redefining the field variable $\left(\rho_{\mathrm{s}} / T\right)^{1 / 2} \varphi=\phi$ we reduce the above Euclidean action to the canonical sine-Gordon form (see, for example, [3]):

$$
S_{\sin }=\int d^{2} x\left[\frac{1}{2}|\nabla \phi-\mathbf{h} \beta / 2 \pi|^{2}+\mu(1-\cos \beta \phi)\right],
$$

with $\beta^{2}=T / \rho_{\mathrm{s}}$ and $\mu=m / T,|\mathbf{h}|=2 \pi|\mathbf{Q}| / \beta^{2}$.

In a similar fashion the sinh-Gordon action becomes

$$
S_{\sinh }=\int d^{2} x\left[\frac{1}{2}|\nabla \phi|^{2}+\mu(\cosh \beta \phi-1)\right] .
$$

For this model there are no kinks and creation of nonzero field gradient would require imaginary field $h$. We do not consider such possibility. 
One can consider the quantum field theory in $(1+1)$-dimensions with $Z=\int \mathcal{D} \phi e^{-S[\phi]}$ where $S$ is given by (3) or (4). The exact solution for both models is known and we can take advantage of the fact that the free energy of a $D$-dimensional classical model is related to the ground state energy $E_{0}$ of the corresponding quantum field theory living in a space of $(D-1)$-dimensions. More specifically, for $D=2$ the partition function of the classical theory defined on a rectangle $L_{x} \times L_{y}$ with periodic boundary conditions in the $x$-direction at temperature $T$ is equal to the partition function of the quantum field theory at temperature $L_{x}^{-1}$ with the coupling constant $\beta^{2}=T$. The limit $L_{x} \rightarrow \infty$ corresponds to the limit of zero temperature in the quantum field theory when its free energy is equal to the ground state energy $E_{0}=L_{y} \mathcal{E}_{0}$. Thus we get the following relation between the free energy per unit area of the 2-dimensional classical model $\mathcal{F}$ and the ground state energy per unit length of the $(1+1)$-dimensional field theory:

$$
\mathcal{F}(T)=T \mathcal{E}_{0}[\beta(T)]
$$

The ground state energy $\mathcal{E}_{0}$ of the quantum sine-Gordon model as a function of parameters $\beta(T)$ and $\mu$ is known exactly ( [3], [4]) and the corresponding expression for the sinh-Gordon model can be extracted from [7].

\section{SINE-GORDON MODEL AT $Q=0$}

We start our discussion of the sine-Gordon model with the case $Q=0$ which is already quite nontrivial. In the Bethe ansatz approach the ground state energy of the quantum sine-Gordon model is calculated by regularizing the model by putting it on a lattice. The lattice constant $a$ and the inverse coupling constant $\theta$ of the regularized model are related to the mass of physical particles. According to [4] the ground state energy $($ at $Q=0)$ is given by:

$$
\mathcal{E}_{0}=\frac{1}{a^{2}} \int_{-\infty}^{+\infty} \frac{\sin 4 \theta t}{t} \frac{\sinh (\pi \tau t)}{\cosh [\pi(1-\tau) t] \sinh (\pi t)} d t
$$

where $\tau=T /\left(8 \pi \rho_{\mathrm{s}}\right) \equiv T / T_{\mathrm{c}}$. The parameters $\theta$ and $a$ are related to the kink's mass: 


$$
m_{\mathrm{s}}=\frac{4}{a} e^{-\theta /(1-\tau)}
$$

To exclude $\theta$ from Eq. (可) we use the T-dependence of the kink's mass given in [3]:

$$
m_{\mathrm{s}}=\frac{1}{a} \frac{2}{\sqrt{\pi}} \frac{\Gamma\left(\frac{1}{2} \frac{T}{T_{\mathrm{c}}-T}\right)}{\Gamma\left(\frac{1}{2} \frac{T_{\mathrm{c}}}{T_{\mathrm{c}}-T}\right)}\left\{\frac{\pi m}{T_{\mathrm{c}}} \frac{\Gamma\left(1-\frac{T}{T_{\mathrm{c}}}\right)}{\Gamma\left(1+\frac{T}{T_{\mathrm{c}}}\right)}\right\}^{\frac{1}{2\left(1-\frac{T}{T_{\mathrm{c}}}\right)}} .
$$

Note that in the limiting case $\tau \ll 1$ we have

$$
m_{\mathrm{s}}=\frac{4}{a} \tau^{-1}\left(m / \pi T_{\mathrm{c}}\right)^{1 / 2}
$$

We should stress that the above expressions make sense only for $m_{\mathrm{s}} a \ll 1$ when the continuous approach works. Therefore to calculate the ground state energy in the continuous approximation in Eq. (6) one has to keep only the pole closest to real axis. Near the point $\tau=1 / 2$ two poles at $t=i$ and $t=i / 2(1-\tau)$ compete and one has to take into account both of them.

Taking this into account we obtain from (6) the general expression for the free energy:

$$
\begin{gathered}
F=F_{1}+F_{2} \\
F_{1}=T \frac{m_{\mathrm{s}}^{2}}{4} \cot \left[\frac{\pi}{2(1-\tau)}\right] \\
=\frac{T}{\pi a^{2}} \cot \left(\frac{\pi}{2\left(1-T / T_{\mathrm{c}}\right)}\right) \frac{\Gamma^{2}\left(\frac{1}{2} \frac{T}{T_{\mathrm{c}}-T}\right)}{\Gamma^{2}\left(\frac{1}{2} \frac{T_{\mathrm{c}}}{T_{\mathrm{c}}-T}\right)}\left\{\frac{\pi m}{T_{\mathrm{c}}} \frac{\Gamma\left(1-\frac{T}{T_{\mathrm{c}}}\right)}{\Gamma\left(1+\frac{T}{T_{\mathrm{c}}}\right)}\right\}^{\frac{1}{\left(1-\frac{T}{T_{\mathrm{c}}}\right)}}, \\
F_{2}=-T \frac{2}{a^{2}}\left(\frac{m_{\mathrm{s}} a}{4}\right)^{4(1-\tau)} \tan [\pi(1-\tau)] .
\end{gathered}
$$

We emphasize that the necessity to keep both terms in the expression for the free energy exists only close to the free fermion point. At $\tau<1 / 2$ the free energy remains finite in the continuous limit $\left(a \rightarrow 0, m_{\mathrm{s}}=\right.$ const $)$, while at $\tau>1 / 2$ it diverges. The latter fact is in agreement with the perturbation theory in $m$ :

$$
\int d^{2} x\langle\cos \beta \phi(x) \cos \beta \phi(0)\rangle \sim \int d^{2} x(x / a)^{-4(1-\tau)}
$$

diverges at small distances. 


\section{FIGURES}

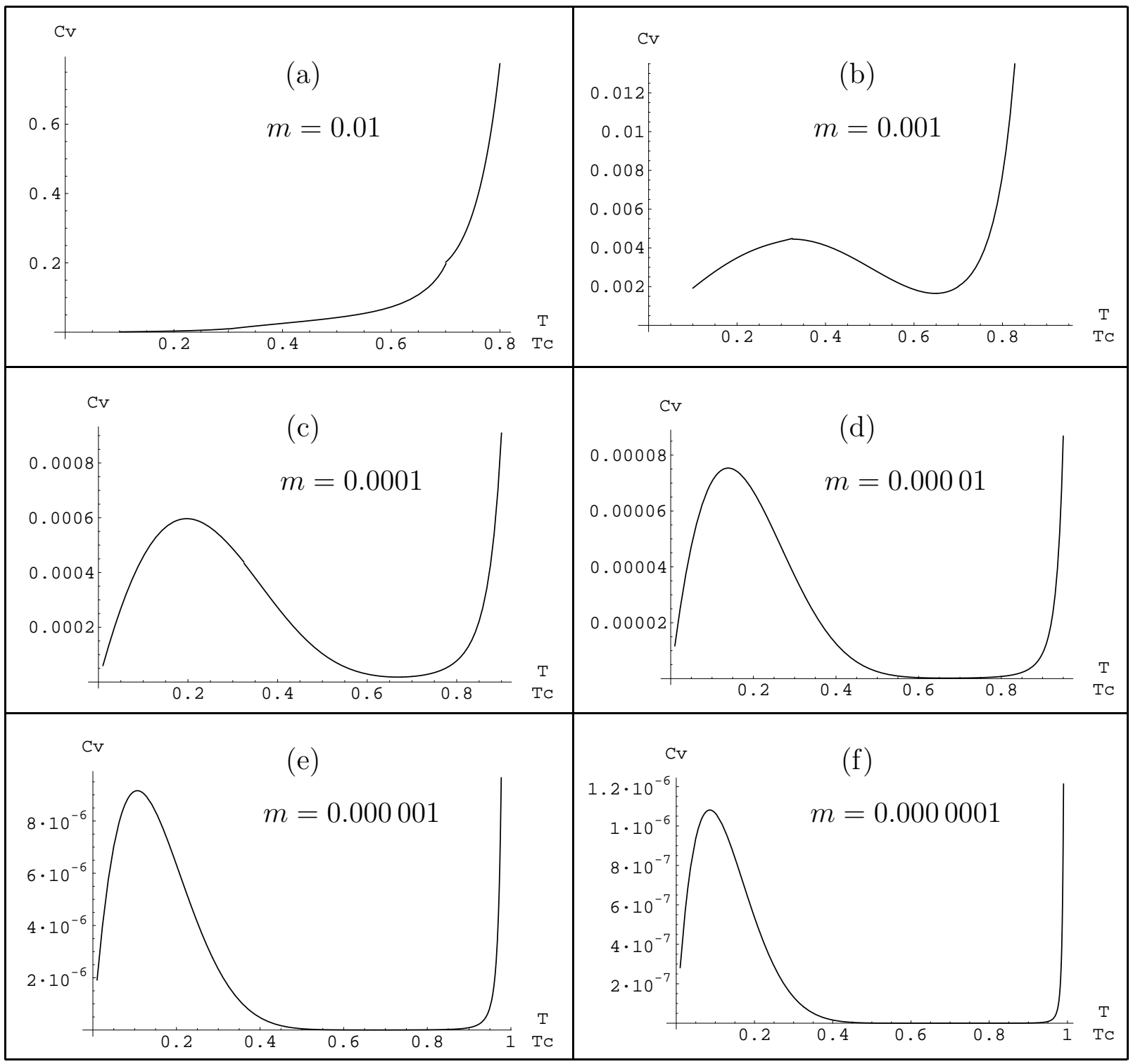

FIG. 1. Plots of the specific heat as a function of $T / T c$, for different values of the parameter $m$, are shown.

Since we always keep a finite, we plot the specific heat at finite values of $\left(\pi m / T_{\mathrm{c}}\right)$, and it is convenient to separate the interval of $\tau$ into three regions. First, for $T / T_{\mathrm{c}} \in(0.1,0.35)$, we take the contribution of only the nearest pole $i / 2(1-\tau)$. Second, the region $T / T_{\mathrm{c}} \in(0.35,0.7)$ where we take the contribution of both poles. Third, for $T / T_{\mathrm{c}} \in(0.7,0.9)$ we take only the contribution of the pole $t=i$.

We combine these results to find the specific heat $C_{\mathrm{v}}(T)=-T \partial^{2} F / \partial^{2} T$ as a function of 


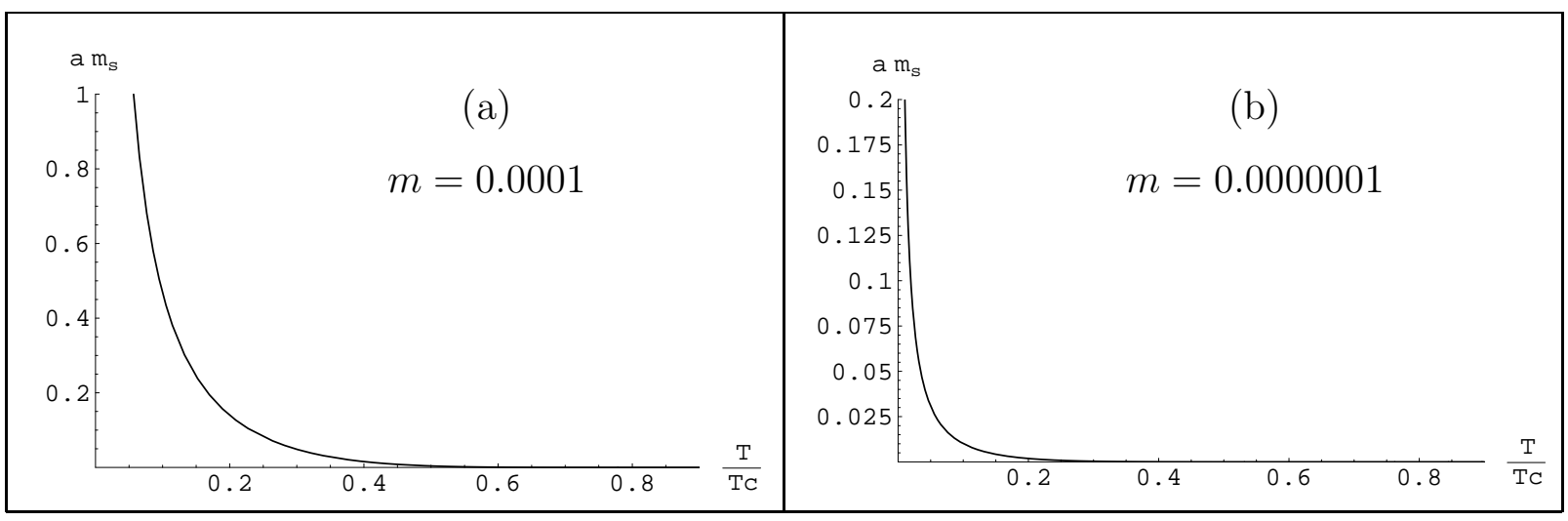

FIG. 2. Plots of $\left(a m_{\mathrm{s}}\right)$ as function of $(T / T c)$, for different values of the parameter $m$, are shown. The condition of continuous approach works on the region $\left(a m_{\mathrm{s}}\right) \ll 1$.

$T / T_{\mathrm{c}}$ and $m / T_{\mathrm{c}}$, in $T / T_{\mathrm{c}} \in(0,1)$. On Fig. 1 we present plots of the temperature of the specific heat for various values of $m$. Fig. 2 gives temperature dependence of the kink's mass. From this picture one one can estimate the region where the condition $m_{\mathrm{s}} a \ll 1$ is fulfilled.

At $1-\tau \ll 1$ and at $\tau \ll 1$ expressions (10) for the specific heat simplify. In the first case we have

$$
\begin{gathered}
a^{2} F=-\frac{2 \pi^{3} m^{2}}{T_{\mathrm{c}}}\left\{\frac{1}{1-\tau}+2 \ln \left[\frac{e^{2}}{\pi(1-\tau)}\right]+O\left((1-\tau) \ln ^{2}(1-\tau)\right)\right\}, \\
C_{\mathrm{v}}=\frac{4 \pi^{3}}{a^{2}} \frac{m^{2} T_{\mathrm{c}}}{\left(T_{\mathrm{c}}-T\right)^{3}}+\ldots .
\end{gathered}
$$

This singularity is associated with the Kosterlitz-Thouless transition at $T=T_{\mathrm{c}}$.

At $\tau \ll 1$ we have

$$
\begin{aligned}
a^{2} F & =-4 \pi^{2} m\left(\frac{\pi m}{T_{\mathrm{c}}}\right)^{1 /(1-\tau)}, \\
C_{\mathrm{v}} & =4 \pi\left(\frac{\pi m}{T_{\mathrm{c}}}\right)^{2} \ln \left(\frac{T_{\mathrm{c}}}{\pi m}\right)\left[2+\ln \left(\frac{T_{\mathrm{c}}}{\pi m}\right)\right] \tau \exp \left\{-\tau \ln \left(\frac{T_{\mathrm{c}}}{\pi m}\right)\right\} .
\end{aligned}
$$

The latter expression explains the existence of the maximum in the specific heat: at $\ln \left(T_{\mathrm{c}} / \pi m\right) \gg 1$ the maximum occurs at $\tau^{*}=\left[\ln \left(T_{\mathrm{c}} / \pi m\right)\right]^{-1}$. 


\section{SINE-GORDON MODEL IN THE INCOMMENSURATE PHASE}

At

$$
Q>Q_{\mathrm{c}}=4 \tau m_{\mathrm{s}}(\tau)
$$

the sine-Gordon model is in the incommensurate phase characterized by a condensate of kinks $\langle\mathbf{Q} \nabla \phi\rangle \neq 0$. The ground state energy of the corresponding quantum field theory acquires an

additional contribution originating from the condensate. The corresponding change in the free energy of the classical model is

$$
\delta F=\frac{\rho_{\mathrm{c}} Q^{2}}{2}+\frac{T m_{\mathrm{s}}}{2 \pi} \int_{-B}^{B} d \theta \cosh \theta \epsilon(\theta)
$$

The nonpositive function $\epsilon(\theta)$ is defined inside the interval $-B<\theta<B$ and satisfies the integral equation (see, for example [3])

$$
\epsilon(\theta)+\int_{-B}^{B} d \theta^{\prime} K\left(\theta-\theta^{\prime}\right) \epsilon\left(\theta^{\prime}\right)=m_{\mathrm{s}} \cosh \theta-\frac{Q}{4 \tau}
$$

where the Fourier image of the kernel is

$$
K(\omega)=\frac{\sinh \frac{\pi(1-2 \tau) \omega}{2(1-\tau)}}{2 \cosh (\pi \omega / 2) \sinh \frac{\pi \tau \omega}{2(1-\tau)}} .
$$

The kernel $K(\theta)$ encodes the information about the soliton-soliton scattering. The limit $B$ is determined by the condition $\epsilon( \pm B)=0$. A possibility of $\epsilon$ being negative appears when the right hand side becomes not positively defined which corresponds to condition (16).

We have solved the integral equation for the function $\epsilon(\theta)$ numerically and the plots of its dependence on $\tau$ and $Q$ are shown in Fig. 4 . The dependence of the limit $B$ on the temperature and $Q$ is shown on Fig. 5 . 


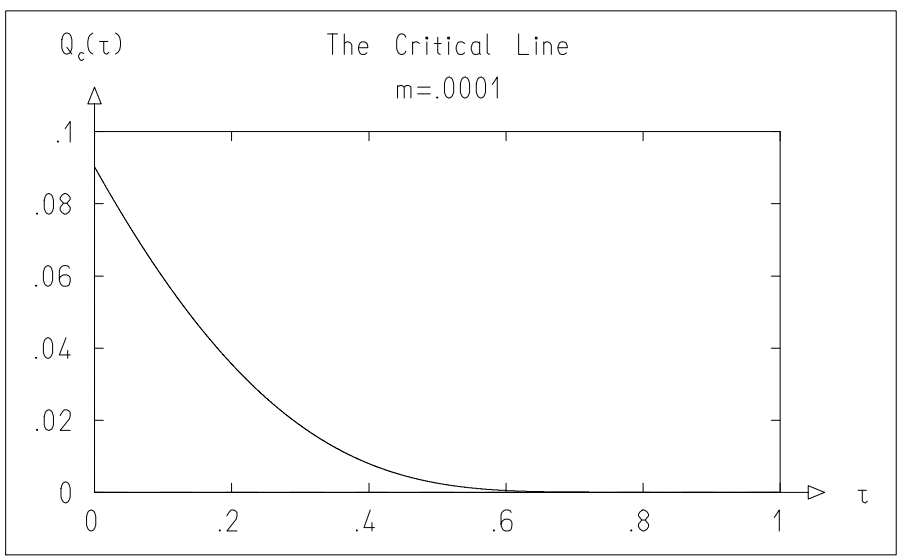

FIG. 3. The critical line as function of $\left(T / T_{\mathrm{c}}\right)$ for a fixed value of the parameter $m$ is shown. The lattice constant $a$ is taken to be unity.

It is curious that the critical field has a finite limit at $\tau \rightarrow 0$ (this limit was first considered in [6]). According to Eq. (9), we have

$$
a Q_{\mathrm{c}}(0)=16\left(\frac{m}{\pi T_{\mathrm{c}}}\right)^{1 / 2}
$$

In the vicinity of the critical line in the incommensurate phase one can expand solution of Eq. (18) in series in $B$ and get for the additional free energy:

$$
\begin{aligned}
\frac{1}{T_{\mathrm{c}}} \delta F= & \frac{Q^{2}}{2} \\
& -\frac{\tau m_{\mathrm{s}}^{2}}{6 \pi}\left(\frac{Q}{Q_{\mathrm{c}}}-1\right)^{3 / 2}\left[1-K(0)\left(\frac{Q}{Q_{\mathrm{c}}}-1\right)^{1 / 2}+\left(0.1+7 \frac{K^{2}(0)}{6}\right)\left(\frac{Q}{Q_{\mathrm{c}}}-1\right)+\ldots\right] .
\end{aligned}
$$

At small $\tau, K(0) \approx \frac{1}{\pi^{2} \tau} \ln (1 / \tau)$ and the expansion is valid for

$$
\pi^{2} \tau / \ln (1 / \tau) \gg\left(Q / Q_{\mathrm{c}}-1\right)
$$

Plots of the additional specific heat are shown on Fig. 6a), whereas plots of the total specific heat are shown in Fig. 6b) for some values of the field $Q$.

The $Q / \tau \rightarrow \infty$ analytic structure of the total free energy $F(Q)$ is given by Zamolodchikov 3

$$
F(Q)-F(0)=\frac{\rho_{\mathrm{c}} Q^{2}}{2}-T \frac{m_{\mathrm{s}}^{2}}{4} \cot \left[\frac{\pi}{2(1-\tau)}\right]-\left(\frac{Q}{4 \tau}\right)^{2} \frac{k(Q / 4 \tau)}{\pi}
$$

The factor $k(Q / 4 \tau)$ is given as a power series 


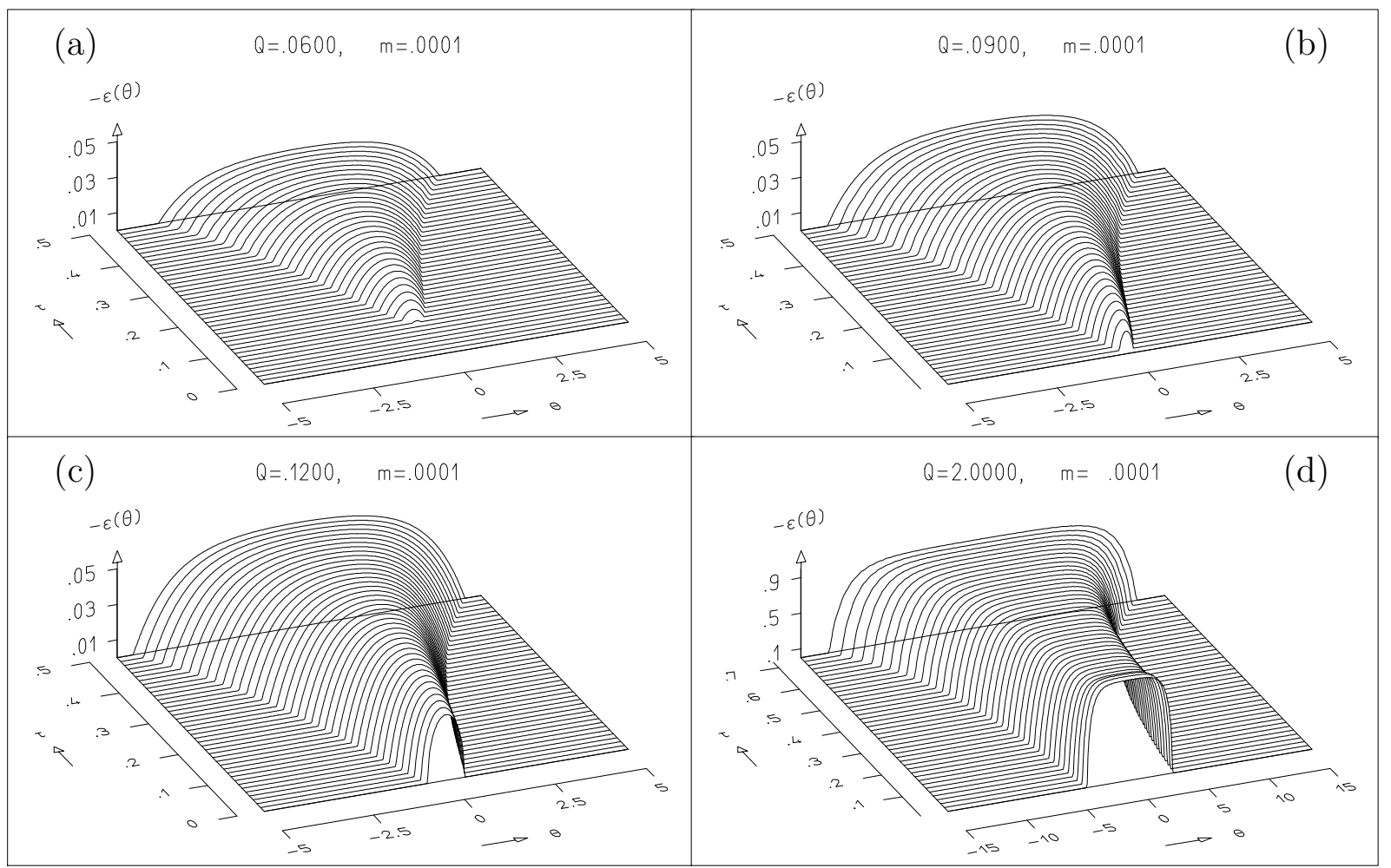

FIG. 4. (a) Plots of $(-\epsilon(\theta))$ for different fixed $\tau$. On figure (b) the parameter $Q=0.090$, which corresponds to crossing the critical line at $\tau \approx 0$.

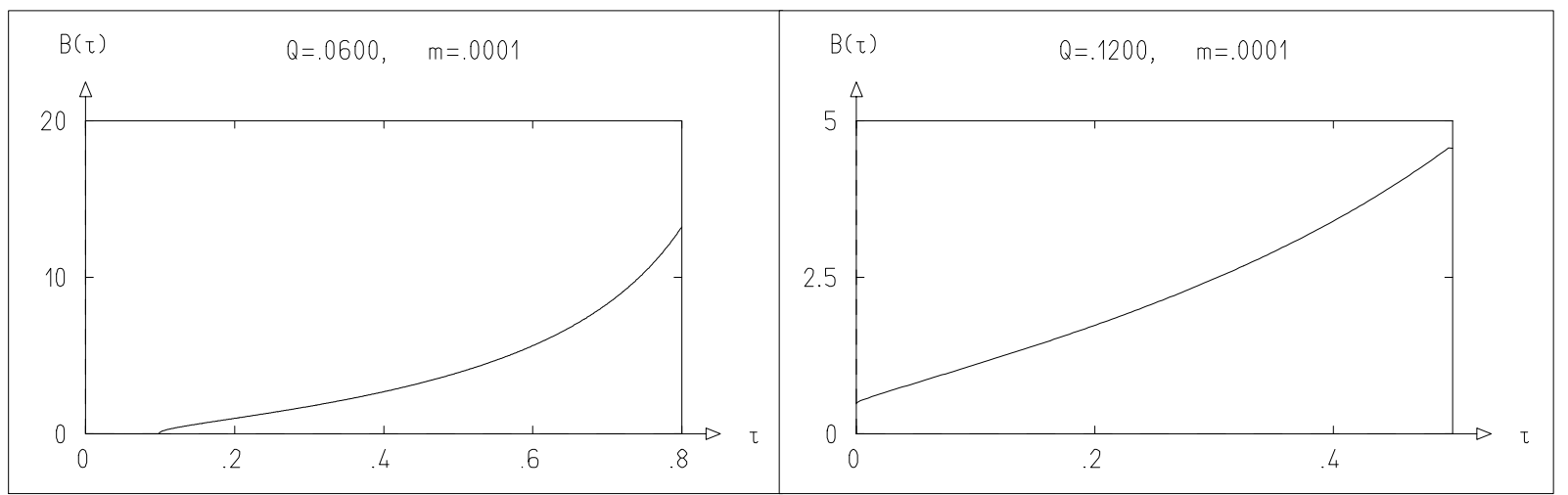

FIG. 5. Plots of temperature dependence of the parameter $B$ in Eq. (18). The parameter $Q$ is taken to have values 0.060 and 0.120 , respectively.

$$
k(Q / 4 \tau)=\sum_{n=0}^{\infty} K_{\mathrm{n}} y^{n}
$$

with

$$
y=\left[\frac{2 m_{\mathrm{s}} \sqrt{\pi}}{Q} \frac{\Gamma\left(\frac{1}{2(1-\tau)}\right)}{\Gamma\left(\frac{\tau}{2(1-\tau)}\right)}\right]^{4(1-\tau)}
$$




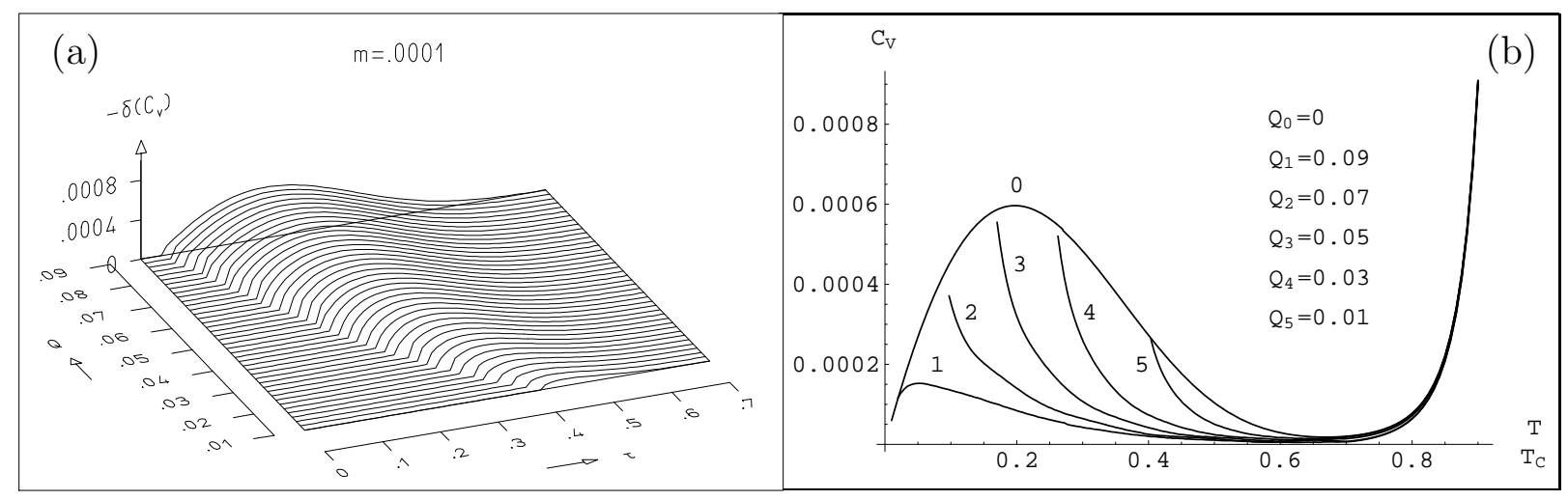

FIG. 6. (a) Three dimensional plot of the contribution to the specific heat coming from the soliton condensate as function of $\tau$ and $Q$. The presence of the condensate leads to decrease in the specific heat. (b) The total specific heat of the system for different values of the parameter $Q$. Each of the lines 1-5 merges with the line $0(Q=0)$ at the commensurate-incommensurate transition temperature.

The first two coefficients are given by

$$
K_{0}=\tau \quad, \quad K_{1}=2 \frac{\Gamma(\tau)}{\Gamma(-\tau)} \frac{\Gamma(5 / 2-\tau)}{\Gamma(1 / 2+\tau)} \frac{\tau}{(2 \tau-1)(3-2 \tau)} .
$$

Putting them together in Eq. (22) we get

$$
\begin{aligned}
F(Q)-F(0)= & \frac{\rho_{\mathrm{c}} Q^{2}}{2}-T \frac{m_{\mathrm{s}}^{2}}{4} \cot \left[\frac{\pi}{2(1-\tau)}\right]-T\left(\frac{Q}{4 \tau}\right)^{2} \frac{\tau}{\pi} \\
& -T \frac{2}{\pi}\left(\frac{Q}{4 \tau}\right)^{2} \frac{\Gamma(\tau)}{\Gamma(-\tau)} \frac{\Gamma(5 / 2-\tau)}{\Gamma(1 / 2+\tau)} \frac{\tau}{(2 \tau-1)(3-2 \tau)}\left[\frac{2 m_{\mathrm{s}} \sqrt{\pi}}{Q} \frac{\Gamma\left(\frac{1}{2(1-\tau)}\right)}{\Gamma\left(\frac{\tau}{2(1-\tau)}\right)}\right]^{4(1-\tau)} .
\end{aligned}
$$

The second term on the right hand side of the above equation gives the free energy of the system in absence of the field $\mathbf{Q}$ (with opposite sign) and cancels with $F(0)$ of the left hand side. On the other hand the first and the third terms on the right cancel each other (keeping in mind that $T_{\mathrm{c}}=8 \pi \rho_{\mathrm{c}}$ ). The total free energy of the system in the presence of the field $\mathbf{Q}$ is given, to this order of approximation, by:

$$
\begin{aligned}
F(Q) & =-T \frac{2}{\pi}\left(\frac{Q}{4 \tau}\right)^{2} \frac{\Gamma(\tau)}{\Gamma(-\tau)} \frac{\Gamma(5 / 2-\tau)}{\Gamma(1 / 2+\tau)} \frac{\tau}{(2 \tau-1)(3-2 \tau)}\left[\frac{2 m_{\mathrm{s}} \sqrt{\pi}}{Q} \frac{\Gamma\left(\frac{1}{2(1-\tau)}\right)}{\Gamma\left(\frac{\tau}{2(1-\tau)}\right)}\right]^{4(1-\tau)} \\
& =-Q^{-2+4 \tau} \frac{T_{\mathrm{c}}}{8 \pi} \frac{\Gamma(\tau)}{\Gamma(-\tau)} \frac{\Gamma(5 / 2-\tau)}{\Gamma(1 / 2+\tau)} \frac{1}{(2 \tau-1)(3-2 \tau)}\left[2 m_{\mathrm{s}} \sqrt{\pi} \frac{\Gamma\left(\frac{1}{2(1-\tau)}\right)}{\Gamma\left(\frac{\tau}{2(1-\tau)}\right)}\right]^{4(1-\tau)} .
\end{aligned}
$$


The expansion is valid only for small values of $\tau<1 / 2$, where the free energy of the system in absence of the field $Q$ is given by $F_{1}$ of Eq. (10).

\section{SINH-GORDON MODEL; THERMODYNAMIC INSTABILITY}

Now we consider the sinh-Gordon model. Here the exact solution was suggested by Fateev [7] who has taken the sine-Gordon two-body S-matrix for the first breathers and changed the sign of the coupling constant $\beta^{2}$ in it. Comparing Eqs.(3), 4) we see that to get the sinh-Gordon action out of the sine-Gordon one we have to change $\beta \rightarrow i \beta$ and $m \rightarrow-m$.

Doing this substitution in the expression for the free energy (see also [7]), we get

$$
\begin{gathered}
F=-m I\left(T / T_{\mathrm{c}}\right)\left(1+T / T_{\mathrm{c}}\right)\left[\frac{T_{\mathrm{c}} \Gamma\left(1-T / T_{\mathrm{c}}\right)}{\pi m \Gamma\left(1+T / T_{\mathrm{c}}\right)}\right]^{\frac{T}{T+T_{\mathrm{c}}}}, \\
I(x)=\exp \left\{2 \int_{0}^{\infty} \frac{d t}{t}\left[-\frac{\cosh ^{2}(x t) \sinh (x t)}{\sinh t \cosh ((1+x) t)}+x e^{-2 t}\right]\right\},
\end{gathered}
$$

where $T_{\mathrm{c}}=8 \pi \rho_{\mathrm{s}}$. This expression is valid for $T<T_{\mathrm{c}}$. The integral (29) can be calculated to give the expression

$$
I(x)=\frac{\Gamma\left(\frac{1}{2}+\frac{x}{2(1+x)}\right)}{\Gamma\left(\frac{1}{2}-\frac{x}{2(1+x)}\right)} \frac{\Gamma(x)}{\Gamma(-x)} \frac{\Gamma\left(-\frac{x}{2(1+x)}\right)}{\Gamma\left(\frac{x}{2(1+x)}\right)},
$$

with $0<x<1$. $I(x)$ is monotonically decreasing function taking values in the interval $(0,1)$.

In the light of the following discussion it will be instructive also to have the expression for the mass (the inverse correlation length) of the sinh-Gordon theory. To get this expression one has to change $\beta$ to $i \beta$ for the sine-Gordon mass which corresponds to reversal of the sign of $T_{\mathrm{c}}$ in Eq.(8):

$$
m_{\mathrm{s}} a=\frac{4 \sqrt{\pi}}{\Gamma\left(\frac{T_{\mathrm{c}}}{2\left(T+T_{\mathrm{c}}\right)}\right) \Gamma\left(1+\frac{T}{2\left(T+T_{\mathrm{c}}\right)}\right)}\left[\frac{\pi m}{T_{\mathrm{c}}} \frac{\Gamma\left(1+T / T_{\mathrm{c}}\right)}{\Gamma\left(1-T / T_{\mathrm{c}}\right)}\right]^{\frac{T_{\mathrm{c}}}{2\left(T+T_{\mathrm{c}}\right)}} .
$$

(This expression coincides with the mass of the sine-Gordon breather after the substitution $T \rightarrow-T)$. We would like to attract the reader's attention to the fact that $m(T)$ never diverges at $T<T_{\mathrm{c}}$ and actually goes to zero at $T \rightarrow T_{\mathrm{c}}$. 
For $\tau=\left(1-T / T_{\mathrm{c}}\right) \ll 1$ we obtain from (29) $I(\tau) \sim \tau$ and substituting this into Eq. (28) we get at $\ln \left(T_{\mathrm{c}} / \pi m\right) \gg 1$

$$
F \sim-\left(1-T / T_{\mathrm{c}}\right)^{1 / 2} e^{-\left(1-T / T_{\mathrm{c}}\right) \ln \left(T_{\mathrm{c}} / \pi m\right)}
$$

It is easy to see that the specific heat becomes negative at

$$
\left(1-T / T_{\mathrm{c}}\right)<(1+\sqrt{2})\left[2 \ln \left(T_{\mathrm{c}} / \pi m\right)\right]^{-1},
$$

such that and at temperatures sufficiently close to $T_{\mathrm{c}}$ the model is thermodynamically unstable.

This thermodynamic instability is not completely unexpected. It occurs in the strong coupling regime when the coupling constant $T / T_{\mathrm{c}}$ approaches its critical value 1 . Let the reader recall that at this value of the coupling constant the ultraviolet limit of the sinhGordon model - the Liouville model becomes unstable [8] (its central charge approaches the value of 25). Another indication of the instability comes from the temperature dependence

of the inverse correlation length given by Eq. (31): at $T \rightarrow T_{\mathrm{c}}$ the mass becomes zero which one does not expect to happen to the sinh-Gordon model which can be thought as the Gaussian theory perturbed by a strongly relevant operator. One can check that at the instability point (33) the mass of the sinh-Gordon particle is still much larger than its value at $T=0$.

\section{CONCLUSIONS}

The summary of our results on the classical sine-Gordon model is well represented by Fig. 6. The specific heat has a peak well below the Kosterlitz-Thouless transition and the temperature dependence becomes even more complicated in the incommensurate phase. We suppose that all these features are detectable experimentally in the relevant systems like the one described in [2]. 


\section{Acknowledgments}

E. P. would like to thank Prof. H. D. Dahmen for the hospitality he offered during the stay at the University of Siegen, Germany, where part of this work was done. He also would like to thank Dave Allen, Joseph Betouras, Pedro Ferreira and especially Tilo Stroh for many

helpful discussions. A. M. T. is grateful to H. Saleur, F. Smirnov V. Fateev and above all to $\mathrm{Yu} \mathrm{Lu}$ for stimulating discussions and valuable remarks. 


\section{REFERENCES}

[1] V. L. Pokrovskii and A. Talapov, Phys. Rev. Lett. 42, 65 (1979).

[2] C. B. Hanna, A. H. MacDonald, S. M. Girvin, cond-mat/9805259, Proceedings of the 12th International Conference on the Electronic Properties of Two-Dimensional Electron Systems (EP2DS-12), Physica B (1998).

[3] A. B. Zamolodchikov, Int. J. Mod. Phys. A10, 1125 (1995).

[4] C. Destri and H. de Vega, Nucl. Phys. B358, 251 (1991).

[5] S. Lukyanov, A. Zamolodchikov, Nucl. Phys. B493, 571 (1997).

[6] M. D. Johnson, N-N. Chen and M. Fowler, Phys. Rev. B34, 7851 (1986).

[7] V. Fateev, S. Lukyanov, A. Zamolodchikov and Al. Zamolodchikov, Phys. Lett. B406, $83(1997)$

[8] A. B. Zamolodchikov and Al. B. Zamolodchikov, Nucl. Phys. B477, 577 (1996). 